\title{
Dicke Simulators with Emergent Collective Quantum Computational Abilities
}

\author{
Pietro Rotondo, ${ }^{1}$ Marco Cosentino Lagomarsino, ${ }^{2}$ and Giovanni Viola ${ }^{3,4}$ \\ ${ }^{1}$ Dipartimento di Fisica, Università degli Studi di Milano and INFN, via Celoria 16, 20133 Milano, Italy \\ ${ }^{2}$ Sorbonne Universités, UPMC Univ Paris 06, UMR 7238, Computational and Quantitative Biology, \\ 15 rue de l'École de Médecine, 75006 Paris, France and CNRS, UMR 7238, 75006 Paris, France \\ ${ }^{3}$ Department of Microtechnology and Nanoscience (MC2), Chalmers University of Technology, SE-412 96 Gothenburg, Sweden \\ ${ }^{4}$ Institute for Quantum Information, RWTH Aachen University, D-52056 Aachen, Germany
}

(Received 26 January 2015; published 6 April 2015)

\begin{abstract}
Using an approach inspired from spin glasses, we show that the multimode disordered Dicke model is equivalent to a quantum Hopfield network. We propose variational ground states for the system at zero temperature, which we conjecture to be exact in the thermodynamic limit. These ground states contain the information on the disordered qubit-photon couplings. These results lead to two intriguing physical implications. First, once the qubit-photon couplings can be engineered, it should be possible to build scalable pattern-storing systems whose dynamics is governed by quantum laws. Second, we argue with an example of how such Dicke quantum simulators might be used as a solver of "hard" combinatorial optimization problems.
\end{abstract}

DOI: 10.1103/PhysRevLett.114.143601

PACS numbers: $42.50 . \mathrm{Pq}, 75.10 . \mathrm{Nr}$

The connection of experimentally realizable quantum systems with computation contains promising perspectives from both the fundamental and the technological viewpoint $[1,2]$. For example, quantum computational capabilities can be implemented by "quantum gates" [3] and by the socalled "adiabatic quantum optimization" technique [4-6]. Today's experimental technology of highly controllable quantum simulators, recently used for testing theoretical predictions in a wide range of areas of quantum physics [7-9], offers new opportunities for exploring computing power for quantum systems.

In the case of light-matter interaction at the quantum level, the reference benchmark is the Dicke model [10]. Studies of its equilibrium properties have predicted a superradiant transition to occur in the strong coupling and low temperature regime [11-13]. The superradiant phase is characterized by a macroscopic number of atoms in the excited state whose collective behavior produces an enhancement of spontaneous emission (proportional to the number of cooperating atoms in the sample). Crucially, this phenomenology is in direct link with experimentally feasible quantum simulators. Recently, Nagy and coworkers [14] argued that the Dicke model effectively describes the self-organization phase transition of a Bose-Einstein condensate in an optical cavity $[15,16]$. Additionally, Dimer and colleagues [17] proposed a cavity-QED realization of the Dicke model based on cavitymediated Raman transitions, closer in spirit to the original Dicke's idea. Evidence of superradiance in this system is reported in [18]. An implementation of generalized Dicke models in hybrid quantum systems has also been put forward [19]. More generally, Dicke-like Hamiltonians describe a variety of physical systems, ranging from circuit
QED [20-24] to cavity QED with Dirac fermions in graphene [25-27]. Additionally, disorder and frustration of the atom-photon couplings have an important role in the study of a Bose-Einstein condensate in multimode cavities $[28,29]$. Recent works [30,31] discussed a multimodalcavity QED simulator with disordered interactions. The authors argue that these systems could be employed to explore spin-glass properties at the quantum level [30-32]. In a follow-up work [33], Gopalakrishnan et al. found an interesting analogy with Hopfield networks at the perturbative level. However, the possible quantum computation applications of this new class of quantum simulators remain relatively unexplored.

In this Letter, we consider a multimode disordered Dicke model with a finite number of modes. We calculate exactly (in the thermodynamic limit) the free energy of the system at temperature $T=1 / \beta$ and we find a superradiant phase transition characterized by the same free-energy landscape of the Hopfield model [34] in the so-called "symmetrybroken" phase, with the typical strong-coupling threshold of the Dicke model. From the theoretical standpoint, our results generalize to the case of quenched disordered couplings the remarkable analysis performed by Lieb et al. [11-13]. The choice of frozen couplings is compatible with the characteristic time scales involved in light-matter interactions. The calculation of the partition function leads us to suggest variational ground states for the model, which we conjecture to be exact in the thermodynamic limit.

The physical consequences of this analysis are fascinating: once the multimode strong-coupling regime is reached and qubit-photon couplings are engineered, it should be possible to build a pattern-storing system whose underlying dynamics is fully governed by quantum laws. Moreover, 
Dicke quantum simulators here analyzed may be suitable to implement specific optimization problems, in the spirit of adiabatic quantum computation [4-6]. We point out a nonpolynomial optimization problem [4,5,35], number partitioning, which could be implemented in a single-mode cavity-QED setup with controllable disorder. Computing applications based on cavity-mediated interactions might own the advantage to be a viable way to generate entangled many-body states with remarkable scalability properties, as recently shown in Ref. [36].

Hopfield's main idea [34] is that the retrieval of stored information, such as memory patterns, may emerge as a collective dynamical property of microscopic constituents ("neurons") whose interconnections ("synapses") are reinforced or weakened through a training phase (e.g., Hebbian learning [37,38]). This is achieved in his model through a fictitious neuronal dynamics whose effect is to minimize the Lyapunov cost function:

$$
E=-\frac{1}{2} \sum_{i, j=1}^{N} T_{i j} S_{i} S_{j}
$$

where $N$ is the number of neurons, $S_{i}=1$ if the $i$ th neuron is active, and -1 otherwise, and the $p$ stored patterns $\xi_{i}^{(k)}=$ $\pm 1 \quad(k=1, \ldots, p)$ determine the interconnections $T_{i j}$ through the relation: $T_{i j}=1 / N \sum_{k} \xi_{i}^{(k)} \xi_{j}^{(k)}-p \delta_{i j}$. The analysis in Ref. [34] shows that the long-time dynamics always converges to one of the $p$ stored patterns; i.e., these configurations are the global minima of the cost function, Eq. (1). The interpretation of this result is that a suitable choice of the interconnections allows us to store a given number of memory patterns into the neural network. Data retrieval is achieved through an algorithm that minimizes the energy function, Eq. (1). A phase transition to a "complex" phase marks the intrinsic limitation on the number of patterns $p$ that can be stored. If $p$ exceeds the critical threshold $p \sim 0.14 N$ many failures in the process of retrieval occur [39,40].

In this Letter, we consider the following multimode Dicke Hamiltonian:

$$
H=\sum_{k=1}^{M} \omega a_{k}^{\dagger} a_{k}+\Delta \sum_{i=1}^{N} \sigma_{i}^{z}+\sum_{i, k=1}^{N, M} \tilde{g}_{i k}\left(a_{k}+a_{k}^{\dagger}\right) \sigma_{i}^{x},
$$

effectively modeling the quantum light-matter interaction of $N$ two-level systems with detuning $\Delta$ and $M$ electromagnetic modes that are supposed to be quasidegenerate at the common frequency $\omega$ and with couplings that we parametrize for future convenience as $\tilde{g}_{i k}=\Omega g_{i k} / \sqrt{N}$, where $\Omega$ is the Rabi frequency and the dimensionless $g_{i k}$ 's are both atom and mode dependent. In cavity-QED realizations, $\omega$ represents the detuning between the cavity frequency and the pumping frequency and could be both positive or negative. A possible choice of the couplings is $g_{i l}=\cos \left(k_{l} x_{i}\right)$, with $k_{l}$ being the wave vector of the photon and $x_{i}$ the position of $i$ th atom [31].

We are interested in the thermodynamic properties of this system in the limit $M \ll N$, and thus in evaluating the partition function $Z=\operatorname{Tr} e^{-\beta H}$. This evaluation can be performed rigorously in the thermodynamic limit $(N \rightarrow \infty)$ using the techniques introduced in Refs. [11-13]. We first consider the fully commuting limit $\Delta=0$. In this case the evaluation of the partition function is straightforward (see Supplemental Material [41]) and we obtain $Z=Z_{\mathrm{FB}} Z_{H}$, where $Z_{\mathrm{FB}}$ is a free boson partition function and $Z_{H}$ is a classical Ising model with a local quenched exchange interactions of the form:

$$
J_{i j}=-\frac{\Omega^{2}}{N} \sum_{k=1}^{M} \frac{g_{i k} g_{j k}}{\omega}
$$

The physical interpretation of this result is that photons mediate long range interactions among the atoms, resulting in an atomic effective Hamiltonian described by a fully connected Ising model (see Fig. 1). The role of the couplings $g_{i k}$ can be understood from Eq. (1) in the context of the Hopfield network. They are the memory patterns stored in the system. By computing exactly the free energy of the model, we will show that this interpretation stays unaltered in the more complicated case $\Delta \neq 0$.

We now proceed to the evaluation of the quantum partition function. We use the method of Wang and co-workers $[13,42]$ (proved to be exact in the

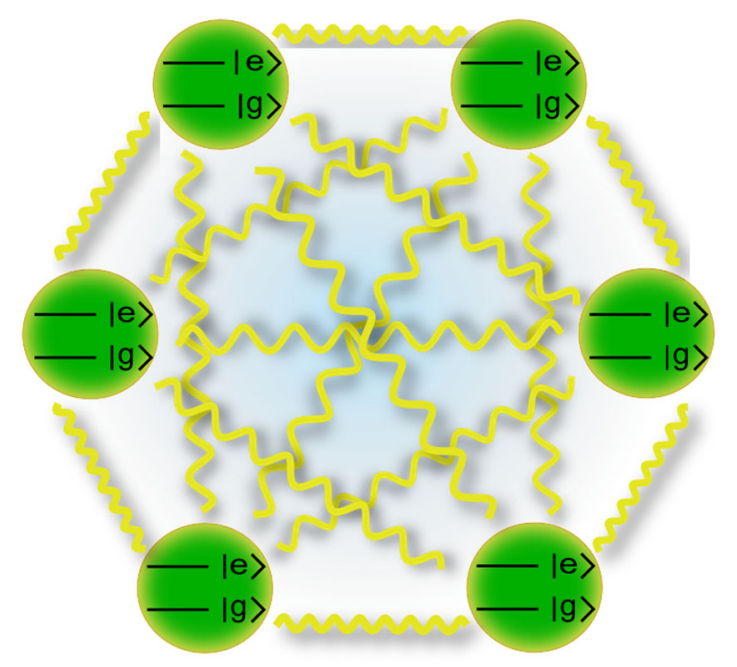

FIG. 1 (color online). In the Dicke model, photons (yellow lines) mediate a long range interaction between qubits (green circles). The drawing sketches schematically a six qubits system within its fully connected graph and its internal level structure. In the standard single-mode Dicke model, the exchange coupling is fixed at the same value for every pair of qubits. In systems where both many modes and disorder are present, the exchange couplings are qubit dependent and take the form given by Eq. (3). 
thermodynamic limit for $M / N \rightarrow 0$ [12]). A generalization of these results to the case of finite-bandwidth frequency is reported in the Supplemental Material [41]. We introduce a set of coherent states $\left|\alpha_{k}\right\rangle$ with $\alpha_{k}=x_{k}+i y_{k}$, one for each electromagnetic mode $k$, and we expand the partition function on this overcomplete basis:

$$
Z=\int \prod_{k=1}^{M} \frac{d^{2} \alpha_{k}}{\pi} \operatorname{Tr}_{A}\left\langle\{\alpha\}\left|e^{-\beta H}\right|\{\alpha\}\right\rangle,
$$

where $\operatorname{Tr}_{A}$ is the atomic trace only. The only technical complication is the calculation of the matrix element in Eq. (4). This turns out to be equal, apart from nonextensive contributions, to the exponential of the operator in Eq. (2) with the replacements $a_{k}, a_{k}^{\dagger} \rightarrow \alpha_{k}, \alpha_{k}^{*}[12,13]$. At this stage the trace over the atomic degrees of freedom can be easily performed. The integral over the imaginary parts of $\alpha_{k}$ 's gives an overall unimportant constant. Finally, defining the $M$-dimensional vectors $\mathbf{x}=\left(x_{1}, x_{2}, \ldots, x_{M}\right)$ and $\mathbf{g}_{i}=\left(g_{i 1}, g_{i 2}, \ldots, g_{i M}\right)$, and with the change of variables $\mathbf{m}=\mathbf{x} / \sqrt{N}$, the partition function assumes a suitable form for performing a saddle-point integration, i.e., $Z=\int d^{M} \mathbf{m} e^{-N f(\mathbf{m})}$. Here, $f$ is the free energy

$$
f(\mathbf{m})=\beta \mathbf{m} \cdot \mathbf{m}-\frac{1}{N} \sum_{i=1}^{N} \log G\left(\mathbf{m}, \mathbf{g}_{i}\right),
$$

with $G\left(\mathbf{m}, \mathbf{g}_{i}\right)=2 \cosh \left[\beta\left(\Delta^{2}+\Omega^{2}\left(\mathbf{g}_{i} \cdot \mathbf{m}\right)^{2}\right)^{\frac{1}{2}}\right]$.

The order parameter $\mathbf{m}$ describes the superradiant phase transition. Physically, it gives the mean number of photons in every mode [43]. Its value is determined by minimizing the free energy in Eq. (5). Solutions of this optimization problem are, in principle, $\mathbf{g}_{i}$ dependent, but in the thermodynamic limit both the free energy and the saddle-point equation are self-averaging [39]. Thus, we conclude that the free energy and the saddle-point equations are given by

$$
\begin{aligned}
f(\mathbf{m}) & =\beta \mathbf{m} \cdot \mathbf{m}-\langle\log G(\mathbf{m}, \mathbf{g})\rangle_{\mathbf{g}}, \\
\mathbf{m} & =\frac{\Omega^{2}}{2}\left\langle\frac{(\mathbf{g} \cdot \mathbf{m}) \mathbf{g}}{\mu(\mathbf{g})} \tanh (\beta \mu(\mathbf{g}))\right\rangle_{\mathbf{g}},
\end{aligned}
$$

with $\mu(\mathbf{g})=\left(\Delta^{2}+\Omega^{2}(\mathbf{g} \cdot \mathbf{m})^{2}\right)^{\frac{1}{2}}$ and $\langle\cdots\rangle_{\mathbf{g}}$ representing the average over the disorder distribution. Equation (6) reduces to the mean-field equations for the Hopfield model for $\Delta \rightarrow 0$ [39]. Thus, $\Delta$ may be intended as a quantum annealer parameter. To fully specify the model, the probability distribution for the couplings is needed. In the following we will assume

$$
P(\mathbf{g})=\prod_{k=1}^{M}\left(\frac{1}{2} \delta\left(g_{k}-1\right)+\frac{1}{2} \delta\left(g_{k}+1\right)\right),
$$

but we have verified that the results are qualitatively robust as long as the disorder is not too peaked around zero in accordance with the classical results of Ref. [39]. To locate the critical point, it suffices to expand in Taylor series Eq. (6). As in the conventional Dicke model, a temperature-independent threshold $\Omega_{c}^{2}=2 \Delta$ emerges. For $\Omega<\Omega_{c}$, the phase transition is inhibited at all temperatures. Whenever the magnitude of the coupling exceeds this threshold value, the critical temperature is located at $T_{c}=\Delta / \operatorname{arctanh}\left(2 \Delta / \Omega^{2}\right)$.

Above the critical temperature $T_{c}$ the only solution to Eq. (6) is a paramagnetic state, with $m_{k}=0$ for all $k$. Below $T_{c}$, different solutions appear. We now set out to classify these solutions and their stability under temperature decrease. For this analysis, we both considered the Hessian matrix $\partial^{2} f / \partial m_{k} \partial m_{l}$ (see Supplemental Material for its explicit expression [41]) and numerical optimization (Fig. 2). The key point, as mentioned above, is that in this symmetry-broken phase the system takes $2 M$ degenerate ground states (as well as many metastable states energetically well separated from the ground states). In other words, also in this fully quantum limit the free-energy landscape still closely resembles that of the Hopfield model [39].

The ground state solutions have the explicit form:

$$
\mathbf{m}_{k}=m^{(1)}(\underbrace{0,0, \ldots, 0}_{k-1 \text { times }}, \underbrace{1,0, \ldots, 0}_{M-k+1 \text { times }}) .
$$

Equation (6) for the order parameter $m^{(1)}$ reduces to $2 \mu\left(m^{(1)}\right)=\Omega^{2} \tanh \left(\beta \mu\left(m^{(1)}\right)\right)$, where $\mu\left(m^{(1)}\right)=$ $\sqrt{\Delta^{2}+\Omega^{2}\left(m^{(1)}\right)^{2}}$. In the zero temperature limit the order parameter can be evaluated exactly:

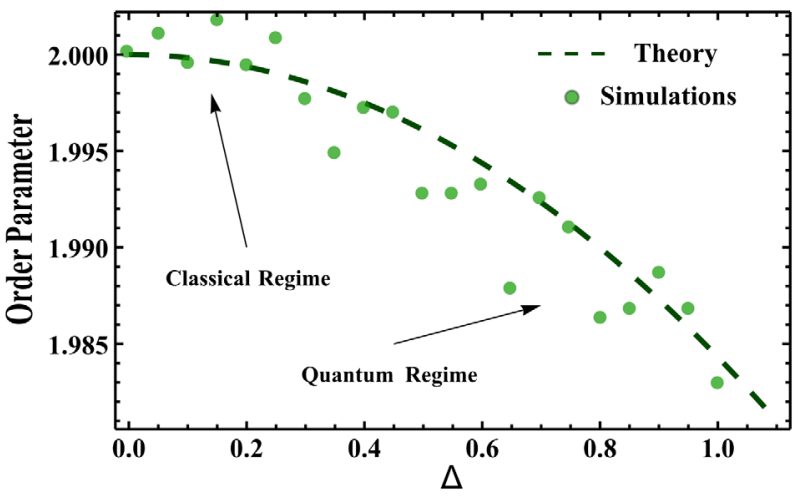

FIG. 2 (color online). Order parameter $m^{(1)}$ in Eq. (9) as a function of $\Delta$ in the ultrastrong coupling regime $\left(\Omega>\Omega_{c}\right)$ at $T=0$. On the theoretical curve (dashed line) are superposed the results (green dots) of a naive numerical optimization algorithm minimizing the ground state energy ansatz in Eq. (11). The parameters of the simulation are $\Omega=4, N=100, M=10$. Green dots are the result of a single realization of the disorder. This plot suggests that at $N=100$ the system is already close to the thermodynamic limit behavior. The classical Hopfield model is recovered in the limit $\Delta \rightarrow 0$. 


$$
m^{(1)}= \pm \sqrt{\frac{\Omega^{2}}{4}-\frac{\Delta^{2}}{\Omega^{2}}} .
$$

At zero temperature the most interesting state is the ground state (GS) of the Hamiltonian equation (2). Inspired by the calculation above we propose the variational ansatz for the GS:

$$
|\mathrm{GS}\rangle=\left|\alpha_{1}, \alpha_{2}, \ldots, \alpha_{M}\right\rangle\left|\operatorname{spin}\left(\alpha_{1}, \ldots, \alpha_{M}\right)\right\rangle,
$$

where $\left|\alpha_{1}, \alpha_{2}, \ldots, \alpha_{M}\right\rangle$ is the product of $M$ coherent states and the spin part is factorized. The mean value of the energy in this GS is given by

$$
E_{\mathrm{GS}}(\mathbf{m})=\mathbf{m} \cdot \mathbf{m}-\left\langle\sqrt{\Delta^{2}+\Omega^{2}(\mathbf{g} \cdot \mathbf{m})^{2}}\right\rangle_{\mathbf{g}}
$$

This expression exactly equals the free energy computed previously in the limit $\beta \rightarrow \infty$, which leads us to conjecture that our factorized variational ansatz is exact. The quantum phase transition is located at the critical coupling $\Omega=\Omega_{c}=\sqrt{2 \Delta}$, at which the paramagnetic solution becomes unstable. In the symmetry-broken phase we have $2 M$ degenerate ground states of the form

$$
|\mathrm{GS}\rangle_{k}=|\underbrace{0,0, \ldots, 0}_{k-1 \text { times }}, \pm m^{(1)}, \underbrace{0, \ldots, 0}_{M-k \text { times }}\rangle\left|\operatorname{spin}\left( \pm m^{(1)}\right)\right\rangle_{k},
$$

with $k=1, \ldots, M$. The spin wave function is also factorized $\left|\operatorname{spin}\left( \pm m^{(1)}\right)\right\rangle_{k}=\prod_{i}\left|s_{i}\right\rangle_{k}$, with

$$
\left|s_{i}\right\rangle_{k}=\frac{1}{\mathcal{N}}\left(-\frac{-\Delta+\sqrt{\Delta^{2}+\beta_{i k}^{2}}}{\beta_{i k}}\left|e_{i}\right\rangle+\left|g_{i}\right\rangle\right),
$$

where $\mathcal{N}$ is a normalization, $\beta_{i k}=g_{i k} m^{(1)}$, and $\left|e_{i}\right\rangle,\left|g_{i}\right\rangle$ are $\sigma_{i}^{z}$ 's eigenstates. It is worth noting that, as expected, the ground state energy is a self-averaging quantity, whereas the ground states are not, being disorder dependent also in the thermodynamic limit.

The above calculation shows that in the superradiant phase, the ground state of the system is a quantum superposition of the $2 M$ degenerate eigenvectors given by Eqs. (12) and (13). Their explicit form suggests that at fixed disorder and mode number the information about the disordered couplings belonging to the $k$ th mode is printed on the atomic wave function. Moreover, the photonic parts of the wave functions are all orthogonal for $k_{1} \neq k_{2}$ in the thermodynamic limit. This implies that, in principle, a suitable measure on the photons subsystem causes the collapse over one of the $2 M$ ground states and gives thus the possibility to retrieve information ("patterns") stored in the atomic wave function.
One may wonder how these ideas may translate in a feasible experimental scheme. As mentioned above, a single-mode Dicke model has been recently realized with cavity-mediated Raman transitions in cavity QED with ultracold atoms [18]. A multimode cavity-QED setup supporting disordered couplings has been proposed in Refs. [30,31], and preliminary evidence of superradiance in this system was found in [44]. Setups operating in a multimode regime were recently suggested also in circuit QED [45-47]. Thus, the ideas on quantum pattern retrieval may directly apply on cavity-QED experimental setups similar to the ones studied in Refs. $[30,33,48]$. The only missing ingredient is a concrete strategy to tune the atomphoton interactions. While this step might be technically involved, single-atom manipulation techniques are rapidly developing [49]. This technology should make it possible to fix the position of the atoms, and thus tune the coupling constants.

We surmise that multimode Dicke quantum setups with controllable disorder could be used beyond storage, to simulate specific optimization problems. Indeed, finding the ground state of classical spin models with disordered interactions is equivalent, in most cases, to finding solutions of computationally expensive nonpolynomial problems [35]. For example, the simplest nonpolynomial-hard problem, number partitioning, could be implemented in a single-mode cavity-QED setup with controllable disorder as follows. Number partitioning can be formulated as an optimization problem [50]: given a set $\mathcal{A}=$ $\left\{a_{1}, a_{2}, \ldots, a_{N}\right\}$ of positive numbers, find a partition, i.e., a subset $\mathcal{A}^{\prime} \subset \mathcal{A}$, such that the residue $E=$ $\left|\sum_{a_{j} \in \mathcal{A}^{\prime}} a_{j}-\sum_{a_{j} \notin \mathcal{A}^{\prime}} a_{j}\right|$ is minimized. A partition can be defined by numbers $S_{j}= \pm 1: S_{j}=1$ if $a_{j} \in \mathcal{A}^{\prime}, S_{j}=-1$ otherwise. The cost function can be replaced by a classical spin Hamiltonian: $H=\sum_{i, j=1}^{N} a_{i} a_{j} S_{i} S_{j}$, whose ground state is equivalent to the minimum partition. In a singlemode cavity-QED network, couplings have the simple form $g_{i}=\cos \left(k x_{i}\right)$ [31]. By the definition of $a=\max _{\mathcal{A}} a_{j}$ and $\tilde{a}_{j}=a_{j} / a$, single-atom manipulation techniques might make it possible to engineer the $g_{i}$ 's in order to implement a given instance of the problem, provided that the cavity is in the "blue" detuned regime and hence the couplings have the appropriate sign [see Eq. (3)]. With a suitably slow annealing of the atomic detuning $\Delta$ (to ensure applicability of the adiabatic theorem), the system should collapse on qubit configurations that are solutions of the corresponding optimization problem.

In conclusion, this Letter provides the first rigorous analysis of the multimode disordered Dicke model, valid beyond the weak-coupling regime and exact in the thermodynamic limit. The equivalence between the multimodal disordered Dicke model and a quantum Hopfield network [51], together with the proposal of a cavity-QED setup implementing a nonpolynomial optimization problem, demonstrates the possibility of quantum computational 
abilities of this new class of quantum simulators. Our proposal is conceptually complementary to a standard quantum computation perspective [52,53]. Indeed, the information can be "written" on the qubits through a quantum annealing on the detuning $\Delta$, similarly to what happens for adiabatic quantum computation [4-6].

We are grateful to B. Bassetti, S. Mandrà, G. Catelani, M. Gherardi, S. Caracciolo, L. Molinari, F. Solgun, and A. Morales for useful discussions and feedback on this manuscript, and to B. Lev for pointing us to useful literature. G. V. was supported by the Alexander von Humboldt foundation and Knut och Alice Wallenbergs foundation.

[1] R. P. Feynman, Int. J. Theor. Phys. 21, 467 (1982).

[2] M. A. Nielsen and I. L. Chuang, Quantum Computation and Quantum Information (Cambridge University Press, Cambridge, 2010).

[3] A. Barenco, C. H. Bennett, R. Cleve, D. P. DiVincenzo, N. Margolus, P. Shor, T. Sleator, J. A. Smolin, and H. Weinfurter, Phys. Rev. A 52, 3457 (1995).

[4] E. Farhi, J. Goldstone, S. Gutmann, J. Lapan, A. Lundgren, and D. Preda, Science 292, 472 (2001).

[5] G. E. Santoro, R. Martoňák, E. Tosatti, and R. Car, Science 295, 2427 (2002).

[6] V. Bapst, L. Foini, F. Krzakala, G. Semerjian, and F. Zamponi, Phys. Rep. 523, 127 (2013).

[7] I. Buluta and F. Nori, Science 326, 108 (2009).

[8] I. M. Georgescu, S. Ashhab, and F. Nori, Rev. Mod. Phys. 86, 153 (2014).

[9] M. Lewenstein, A. Sanpera, V. Ahufinger, B. Damski, A. Sen, and U. Sen, Adv. Phys. 56, 243 (2007).

[10] R. Dicke, Phys. Rev. 93, 99 (1954).

[11] K. Hepp and E. Lieb, Ann. Phys. (N.Y.) 76, 360 (1973).

[12] K. Hepp and E. Lieb, Phys. Rev. A 8, 2517 (1973).

[13] Y. K. Wang and F. T. Hioe, Phys. Rev. A 7, 831 (1973).

[14] D. Nagy, G. Kónya, G. Szirmai, and P. Domokos, Phys. Rev. Lett. 104, 130401 (2010).

[15] K. Baumann, C. Guerlin, F. Brenneke, and T. Esslinger, Nature (London) 464, 1301 (2010).

[16] K. Baumann, R. Mottl, F. Brennecke, and T. Esslinger, Phys. Rev. Lett. 107, 140402 (2011).

[17] F. Dimer, B. Estienne, A. S. Parkins, and H. J. Carmichael, Phys. Rev. A 75, 013804 (2007).

[18] M. P. Baden, K. J. Arnold, A. L. Grimsmo, S. Parkins, and M. D. Barrett, Phys. Rev. Lett. 113, 020408 (2014).

[19] L. J. Zou, D. Marcos, S. Diehl, S. Putz, J. Schmiedmayer, J. Majer, and P. Rabl, Phys. Rev. Lett. 113, 023603 (2014).

[20] Y. Zhang, L. Yu, J.-Q. Liang, G. Chen, S. Jia, and F. Nori, Sci. Rep. 4, 4083 (2014).

[21] P. Nataf and C. Ciuti, Nat. Commun. 1, 72 (2010).

[22] O. Viehmann, J. von Delft, and F. Marquardt, Phys. Rev. Lett. 107, 113602 (2011).

[23] J. A. Mlynek, A. A. Abdumalikov, C. Eichler, and A. Wallraff, Nat. Commun. 5, 5186 (2014).
[24] A. Mezzacapo, U. Las Heras, J. S. Pedernales, L. DiCarlo, E. Solano, and L. Lamata, Sci. Rep. 4, 7482 (2014).

[25] L. Chirolli, M. Polini, V. Giovannetti, and A. H. MacDonald, Phys. Rev. Lett. 109, 267404 (2012).

[26] D. Hagenmüller and C. Ciuti, Phys. Rev. Lett. 109, 267403 (2012).

[27] F. M. D. Pellegrino, L. Chirolli, R. Fazio, V. Giovannetti, and M. Polini, Phys. Rev. B 89, 165406 (2014).

[28] S. Gopalakrishnan, B. Lev, and P. Goldbart, Nat. Phys. 5, 845 (2009).

[29] S. Gopalakrishnan, B. L. Lev, and P. M. Goldbart, Phys. Rev. A 82, 043612 (2010).

[30] S. Gopalakrishnan, B. L. Lev, and P. M. Goldbart, Phys. Rev. Lett. 107, 277201 (2011).

[31] P. Strack and S. Sachdev, Phys. Rev. Lett. 107, 277202 (2011).

[32] P. Rotondo, E. Tesio, and S. Caracciolo, Phys. Rev. B 91, 014415 (2015).

[33] S. Gopalakrishnan, B. L. Lev, and P. M. Goldbart, Philos. Mag. 92, 353 (2012).

[34] J. J. Hopfield, Proc. Natl. Acad. Sci. U.S.A. 79, 2554 (1982).

[35] A. Lucas, Front. Phys. 2, 5 (2014).

[36] C. Aron, M. Kulkarni, and H. E. Türeci, arXiv:1412.8477.

[37] D. O. Hebb, Arch. Neurol. Psychiatry 44, 421 (1940).

[38] D. O. Hebb, in Brain Mechanisms and Learning, edited by J.F. Delafresnaye (Oxford University Press, London, 1961).

[39] D. J. Amit, H. Gutfreund, and H. Sompolinsky, Phys. Rev. A 32, 1007 (1985).

[40] D. J. Amit, H. Gutfreund, and H. Sompolinsky, Phys. Rev. Lett. 55, 1530 (1985).

[41] See Supplemental Material at http://link.aps.org/ supplemental/10.1103/PhysRevLett.114.143601 for the derivation of Equations (3) and (5) and for the analysis of the finite bandwidth case.

[42] J. Larson and M. Lewenstein, New J. Phys. 11, 063027 (2009).

[43] C. Emary and T. Brandes, Phys. Rev. Lett. 90, 044101 (2003).

[44] A. Wickenbrock, M. Hemmerling, G. R. M. Robb, C. Emary, and F. Renzoni, Phys. Rev. A 87, 043817 (2013).

[45] D. J. Egger and F. K. Wilhelm, Phys. Rev. Lett. 111, 163601 (2013).

[46] M. Leib and M. J. Hartmann, Phys. Rev. Lett. 112, 223603 (2014).

[47] T. H. Kyaw, D. A. Herrera-Martí, E. Solano, G. Romero, and L.-C. Kwek, Phys. Rev. B 91, 064503 (2015).

[48] A. J. Kollár, A. T. Papageorge, K. Baumann, M. A. Armen, and B. L. Lev, arXiv:1411.5443.

[49] F. Nogrette, H. Labuhn, S. Ravets, D. Barredo, L. Béguin, A. Vernier, T. Lahaye, and A. Browaeys, Phys. Rev. X 4, 021034 (2014).

[50] S. Mertens, Phys. Rev. Lett. 81, 4281 (1998).

[51] Y. Nonomura and H. Nishimori, arXiv:cond-mat/9512142.

[52] D. Loss and D. P. DiVincenzo, Phys. Rev. A 57, 120 (1998).

[53] R. Raussendorf, D. E. Browne, and H. J. Briegel, Phys. Rev. A 68, 022312 (2003). 